\title{
The biological effects of high-pressure gas on the yeast transcriptome
}

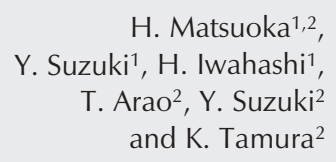

H. Matsuoka ${ }^{1,2}$,

Y. Suzuki ${ }^{1}$, H. Iwahashi',

T. Arao ${ }^{2}$, Y. Suzuki ${ }^{2}$

and K. Tamura ${ }^{2}$

\author{
${ }^{1}$ National Institute of Advanced Industrial Science and Technology, \\ Tsukuba, Ibaraki, Japan \\ 2Department of Chemical Science and Technology, Faculty of Engineering, \\ The University of Tokushima, Minamijosanjima-cho, Tokushima, Japan
}

\section{Correspondence \\ H. Matsuoka \\ National Institute of Advanced Industrial Science and Technology \\ Central 6, Higashi 1-1-1 \\ Tsukuba, Ibaraki 305-8566 \\ Japan \\ E-mail: hitoshi.iwahashi@aist.go.jp \\ Presented at the 3rd International Conference on High Pressure Bioscience and Biotechnology, Rio de Janeiro, RJ, Brazil, September 27-30, 2004.}

Received January 21, 2005 Accepted June 21, 2005

\begin{abstract}
The aim of the present study was to examine the feasibility of DNA microarray technology in an attempt to construct an evaluation system for determining gas toxicity using high-pressure conditions, as it is well known that pressure increases the concentration of a gas. As a first step, we used yeast (Saccharomyces cerevisiae) as the indicator organism and analyzed the mRNA expression profiles after exposure of yeast cells to nitrogen gas. Nitrogen gas was selected as a negative control since this gas has low toxicity. Yeast DNA microarray analysis revealed induction of genes whose products were localized to the membranes, and of genes that are involved in or contribute to energy production. Furthermore, we found that nitrogen gas significantly affected the transport system in the cells. Interestingly, nitrogen gas also resulted in induction of cold-shock responsive genes. These results suggest the possibility of applying yeast DNA microarray to gas bioassays up to $40 \mathrm{MPa}$. We therefore think that "bioassays" are ideal for use in environmental control and protection studies.
\end{abstract}

\section{Introduction}

While seeking a comfortable living environment, the human race has been continuously releasing large quantities of pollutants into the environment, consequently causing various problems such as air, water, and soil pollution. Although emission of pollutants is being gradually reduced by the recent tighter control of emissions of environmental pollutants, we are still polluting our environment. The problem is further compounded by the fact that we are not dealing with a single pollutant, but with a mixture of pollutants in the environment, and therefore it is rather difficult to recognize their effects on organisms. Methods that can determine their effect primarily include physiochemical
Key words

- DNA microarray

- Yeast

- Hydrostatic pressure

- Gas pressure

- Nitrogen gas analyses and bioassays. When pollution is supposed to be present to some extent, we can use physicochemical analyses as a qualitative and quantitative tool for a single substance; however, this is not useful for the assessment of the impact of tens of millions of chemical substances on the environment. Although we cannot determine the concentration of each chemical substance by bioassay, it is possible to do rapid toxicity and genetic toxicity analysis using animal organisms (mammals such as rodents, fish and shellfish, algae, single cells, etc.) because it focuses attention on the effect on organisms.

We have studied a bioassay for the hydrosphere using a yeast DNA microarray. In the present study, drawing on our previous experience, we attempted to develop a bioas- 
say system for polluted air monitoring. By using yeast (Saccharomyces cerevisiae S288C), suitable for rapid toxicity assessment, we can monitor genome-wide gene expression profiles by DNA microarray after exposing yeast cells to high-pressure gas. Thus, we believe it will be possible to assess the impact of polluted gases within short periods of time.

In the present report, we focus mainly on nitrogen gas, a component of our life-supporting air in the environment. Our results revealed significant changes in gene expression profiles, such as those involved in the membrane system, energy, cellular transport, and even cold-response. Since we were able to detect and characterize the response, we conclude that we may use high-pressure gas conditions for gas bioassay up to $40 \mathrm{MPa}$.

\section{Material and Methods}

\section{Strain and growth conditions}

Saccharomyces cerevisiae S288C (suc2 mal gal2 CUP1) cultured in yeast extract/ peptone/dextrose medium (YPD) (2\% polypeptone, $1 \%$ yeast extract, $2 \%$ glucose) were inoculated into fresh YPD in two Erlenmeyer flasks. Yeast cells were grown to an absorbance $\left(\mathrm{A}_{660}\right)$ of 0.9 to 1.0 at $660 \mathrm{~nm}$ at $30^{\circ} \mathrm{C}$ before pressurization.

\section{Pressure treatment}

The cultures were placed in two nylon bags and the holes were sealed with cotton wool, which were transferred to a high-pressure vessel (30-11HF4, High Pressure Equipment Co. Ltd., Elie, PA, USA). The sample was pressurized with nitrogen gas at $40 \mathrm{MPa}$ and a control was kept under atmospheric pressure of $0.1 \mathrm{MPa}$. Both sample and control were incubated in a thermostated bath for $2 \mathrm{~h}$ at $30^{\circ} \mathrm{C}$. Thirty minutes were needed to bring the pressure up to $40 \mathrm{MPa}$, followed by another $30 \mathrm{~min}$ for decompression. Yeast cells were then incubated for $2 \mathrm{~h}$ at $40 \mathrm{MPa}$, thus being exposed to high pressure for a total of $3 \mathrm{~h}$. The control was incubated for 3 $\mathrm{h}$ under the same conditions as the experimental sample, except that the pressure was $0.1 \mathrm{MPa}$.

\section{DNA microarray analysis}

Total RNA was isolated by the hot phenol method (1). Poly(A)+RNA was purified from total RNA with an Oligotex-dT30 mRNA purification kit (Takara, Otsu, Japan). Fluorescently labeled cDNA was synthesized by oligo dT-primer polymerization using PowerScript ${ }^{\mathrm{TM}}$ reverse transcriptase. cDNA obtained from control poly(A)+RNA was fluorescently labeled with $\mathrm{Cy} 3$ and cDNA obtained from the poly(A)+RNA of the sample treated with nitrogen gas was labeled with Cy5. Two to four micrograms poly(A)+RNA was used for each labeling and the same amount of each poly(A)+RNA was used on one slide. The two labeled cDNA pools were mixed and hybridized with a yeast DNA chip (DNA Chip Research, Inc., Yokohama, Japan) for 24-36 h at $65^{\circ} \mathrm{C}$. Open reading frames of 200 - to 8,000-bp DNA (0.1-0.5 ng) were spotted on this microarray, and 5880 genes could be analyzed under these conditions. After hybridization, the labeled microarrays were washed and dried and scanned with a confocal laser Scanarray 4000 system (GSI Lunomics, Billeria, MA, USA). The resulting imaging data were quantified using the Quantarray system (GSI Lunomics). The fluorescence intensity of each spot on the image was subtracted from the background, and the $\mathrm{Cy} 5 / \mathrm{Cy} 3$ intensity ratios were calculated and normalized using the median value of the GeneSpring software (Silicon Genetis, Red Wood, CA, USA) as positive control. The DNA microarray data in this report were obtained from two sheets of DNA microarray images. The details of the microarray procedure have been described previously (2). 


\section{Cluster analysis}

To extract meaningful information from the large amount of data generated by the DNA microarray, we applied a bioinformatics tool called hierarchical cluster analysis which permits one to recognize changes in the transcription patterns following exposure to nitrogen gas. Hierarchical clustering was carried out using GeneSpring (3). The settings for the calculations were as follows: similarity was measured by standard correlation, the separation ratio value was 1.0 , and the minimum distance value was 0.001 . The genes used for this analysis have been described previously (4).

\section{Results and Discussion}

\section{Conditions for treatment with pressurized gas}

In DNA microarray analysis, the most important factor corresponds to the conditions for stress treatment. A weak stress cannot be expected to significantly affect cell physiology, whereas a strong stress may cause cell death. Generally, we can expect sufficient stress responses under a treatment that causes about 50\% growth inhibition (511). After the pressure treatment, colonyforming units were monitored to select the appropriate conditions of pressure treatment. We found that $40 \mathrm{MPa}$ of nitrogen gas caused $50 \%$ growth inhibition and consequently selected this condition for the pressure treatment.

\section{Clustering analysis of hydrostatic pressure and gas pressure}

Hierarchical cluster analysis was performed using the GeneSpring version 4.2.1 software (2). The clustering algorithm arranges conditions according to their similarity in expression profiles across all conditions, in such a way that conditions with similar patterns are clustered together as in a taxonomic tree (Figures 1 and 2). Cluster analysis of high-pressure conditions (hydrostatic pressure and gas pressure), surface

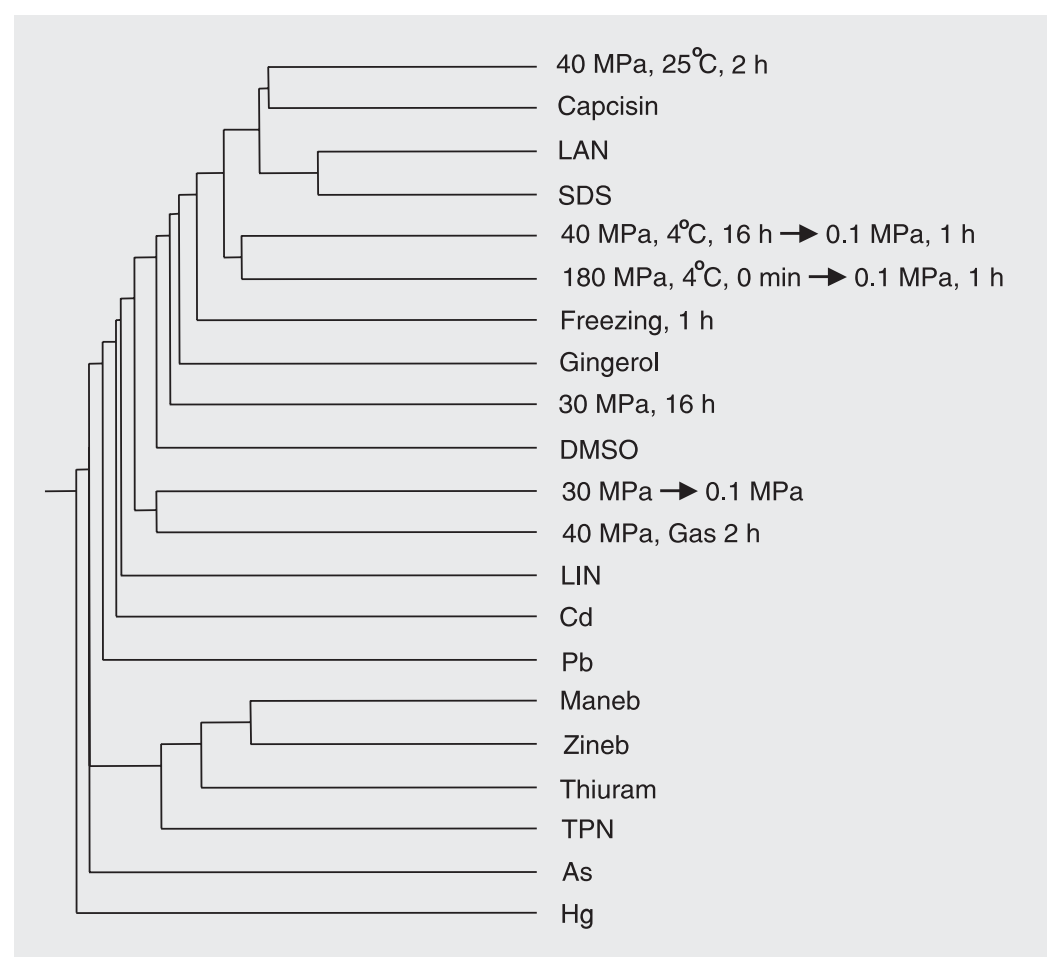

Figure 1. Cluster analysis of expression profiles following treatment of yeast with compressed gas pressures, heavy metals, surface active agent, freezing, and pesticides. Expression profiles based on 6335 spots were used for the calculation.

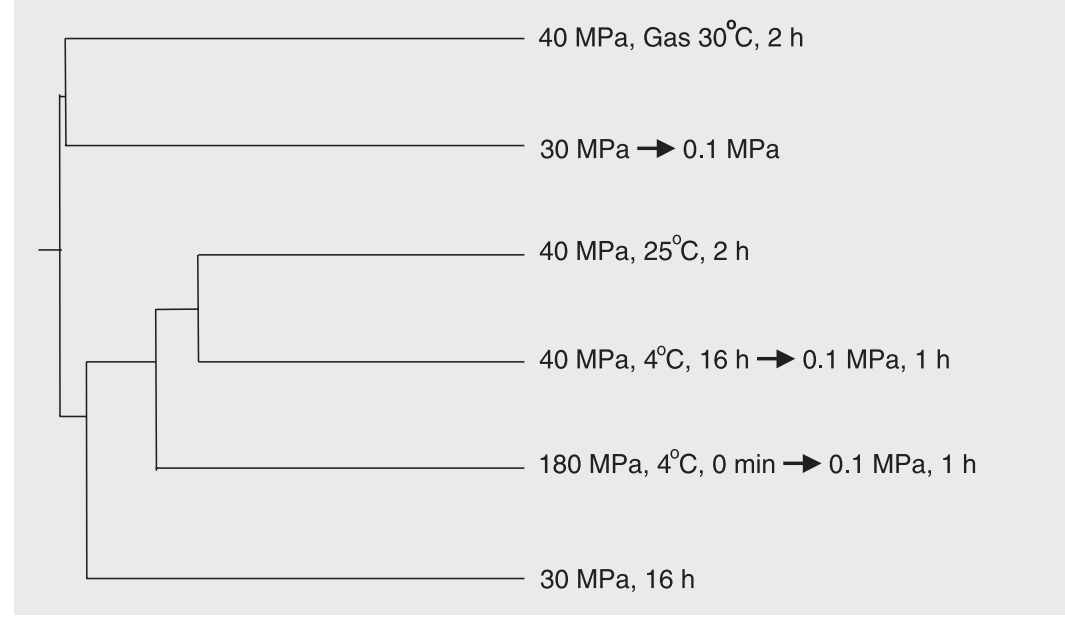

Figure 2. Cluster analysis of expression profiles following treatment of yeast with six different compressed gas pressures. A dendrogram was constructed to indicate the relationship among the effects of each compressed pressure. Expression profiles based on 6335 spots were used for the calculation. 
active agent, heavy metals, freezing, and pesticide chemicals is shown in Figure 1. We found that high pressure (hydrostatic pressure and gas pressure), surface active agent, and freezing were grouped together, and were separate from pesticide chemicals and heavy metals. We also compared nitrogen gas pressure conditions with other pressure conditions (Figure 2). An interesting observation was that a 40-MPa pressure of nitrogen gas at $30^{\circ} \mathrm{C}$ for $2 \mathrm{~h}$ did not form a cluster with hydrostatic pressure of $40 \mathrm{MPa}$ at $25^{\circ} \mathrm{C}$ for $2 \mathrm{~h}$.

\section{Characterization of induced genes by their functions}

It was possible to detect 6335 spots. After global normalization, we removed the spots with a Cy5 intensity of less than 4000 because of the doubtful results of lower intensity. Given that more than $99 \%$ of the control spots without DNA had intensities lower than 1000 for $\mathrm{Cy} 3$, the cutoff line for precluding negative results was taken as a scanned intensity below 2000 for Cy3 (which corresponded to an intensity of 4000 for Cy5) (12). Results between 1000 and 2000 of intensity for $\mathrm{Cy} 3$ were considered doubtful, and therefore discarded. Finally, we selected a total of 5164 genes from 6335 spots and from these 5164 genes we selected 226 as the induced genes and 210 as the repressed genes, by pressure treatment. The 226 induced genes were selected as the genes having a Cy5 intensity two-fold higher than the $\mathrm{Cy} 3$ intensity. The 210 repressed genes were also selected as the genes having a $\mathrm{Cy} 5$ intensity 0.5 times lower than the $\mathrm{Cy} 3$ intensity.

We characterized induced genes using the functional category of MIPS (http:// mips.gsf.de/) from two microarray data sets. MIPS is a database in which all yeast genes are assigned to 18 functional categories and more than 100 subcategories. Using this database we are able to determine the functions of the genes. Table 1 shows the functional

Table 1. List of functional categories and subcategories activated by nitrogen gas pressure.

\begin{tabular}{lrcr}
\hline Functional categories and functional subcategories & $\%$ & No. of genes & Total number \\
\hline Energy & 17.9 & 45 & 252 \\
$\quad$ Respiration & 23.9 & & \\
Transport facilitation & $\mathbf{8 . 6}$ & $\mathbf{2 7}$ & $\mathbf{3 1 3}$ \\
$\quad$ Transport mechanism & 16.2 & & 199 \\
Regulation of/interaction with the cellular environment & $\mathbf{8 . 0}$ & $\mathbf{1 6}$ & 1066 \\
$\quad$ lonic homeostasis & 11.0 & & 278 \\
Metabolism & 5.9 & 63 & 495 \\
Cell rescue, defense and virulence & 5.4 & 15 & 2258 \\
Cellular transport and transport mechanisms & 4.8 & 24 & 115 \\
Subcellular localization & 4.5 & 11 & 359 \\
Classification not yet fully determined & 4.3 & 5 & 209 \\
Protein synthesis & 3.6 & 13 & 595 \\
Control of cellular organization & 2.9 & 6 & 2399 \\
Protein fate (folding, modification, destination) & 2.5 & 15 & 427 \\
Unclassified proteins & 2.1 & 51 & 628 \\
Cell fate & 1.9 & 8 & 771 \\
Cell cycle and DNA processing & 1.8 & 11 & 59 \\
Transcription & 1.6 & 12 & 13 \\
Cellular communication/signal protein activity regulation & 0 & 0 & 116 \\
Transduction mechanism & 0 & 0 & 0 \\
Transposable elements, viral and plasmid proteins & 0 & 0 & \\
\hline
\end{tabular}

The functional categories and the proportion of induced genes in categorized genes are listed. The extensively activated categories are shown in bold. 
characterization of 226 genes that were considered to be the induced genes. The functional categories that were significantly induced were "Energy (17\%)", "Transport facilitation (8.6\%)", and "Regulation of/interaction with cellular environment $(8.0 \%)$ ". Furthermore, the "Energy" category was characterized by the "Respiration" subcategory (Table 1), which in turn was further characterized with genes whose products were localized in mitochondria, such as cytochrome $\mathrm{c}$ oxidases. The categories of
"Transport facilitation" and "Regulation of/ interaction with cellular environment" were characterized by the subcategories of "Transport mechanism" and "Ionic homeostasis" (Table 1). These two subcategories belong to different categories which, however, consist of transporter genes. For example, the $\mathrm{ABC}$ transporter and $\mathrm{Na}^{+}$pump and ATP synthase belong to these subcategories. Table 2 lists the top 25 highly induced genes treated with nitrogen gas under $40 \mathrm{MPa}$ at $30^{\circ} \mathrm{C}$ for $2 \mathrm{~h}$. The genes related to the transport system

Table 2. Top 25 induced open reading frames under pressure of $40 \mathrm{MPa}$ at $30^{\circ} \mathrm{C}$ for $2 \mathrm{~h}$.

\begin{tabular}{|c|c|c|}
\hline Name & Ratio & Description \\
\hline BTN2 & $\underline{20.5}$ & Gene/protein whose expression is elevated in a btn1 \\
\hline YLR162W & 15.2 & Hypothetical protein \\
\hline SCW4 & 14.3 & $\begin{array}{l}\text { Soluble cell wall protein can be released from SDS-extracted cell walls under } \\
\text { reducing conditions }\end{array}$ \\
\hline THI22 & 10.7 & Similarity to Bacillus subtilis transcriptional activator tenA \\
\hline JEN1 & 10.7 & Carboxylic acid transporter protein homolog \\
\hline$\underline{\mathrm{AGP} 1}$ & $\underline{8.8}$ & Amino acid permease \\
\hline ENA1 & $\underline{8.1}$ & Plasma membrane P-type ATPase involved in $\mathrm{Na}^{+}$and $\mathrm{Li}^{+}$efflux \\
\hline TIR1 & 8.1 & Cold-shock-induced protein of the Srp1p, Tip1p family of serine-alanine-rich proteins \\
\hline MSD1 & 7.7 & Aspartyl-tRNA synthetase, mitochondrial \\
\hline ENA5 & $\underline{7.3}$ & Plasma membrane P-type ATPase involved in $\mathrm{Na}^{+}$and $\mathrm{Li}^{+}$efflux \\
\hline$\underline{\text { CTR1 }}$ & $\underline{6.6}$ & Plasma membrane copper transport protein \\
\hline CAT8 & 6.5 & Zinc-cluster protein involved in activating gluconeogenic genes related to Gal $4 p$ \\
\hline FIT2 & $\underline{6.3}$ & $\begin{array}{l}\text { Mannoprotein that is incorporated into the cell wall via aglycosylphosphatidylinositol } \\
\text { anchor, involved in the retention of siderophore iron in the cell wall }\end{array}$ \\
\hline ENA2 & $\underline{6.0}$ & Plasma membrane P-type ATPase involved in $\mathrm{Na}^{+}$and $\mathrm{Li}^{+}$efflux \\
\hline DED1 & 5.9 & ATP-dependent RNA helicase of the DEAD box family \\
\hline$\underline{\mathrm{COX} 3}$ & $\underline{5.6}$ & Cytochrome c oxidase subunit III, mitochondrially coded \\
\hline RPL4A & 5.1 & Ribosomal protein L4A (L2A) (rp2) (YL2) \\
\hline YLR201C & 5.0 & Hypothetical protein \\
\hline$\underline{\mathrm{HXT5}}$ & $\underline{4.9}$ & $\underline{\text { Hexose transporter }}$ \\
\hline$\underline{\operatorname{cox} 2}$ & $\underline{4.8}$ & Subunit II of cytochrome c oxidase \\
\hline ATP1 & $\underline{4.7}$ & Mitochondrial F1F0-ATPase alpha subunit \\
\hline TIR2 & 4.6 & Induced by cold shock \\
\hline TY1A & 4.4 & TyA gag protein. Gag processing produces capsid proteins \\
\hline SPI1 & 4.4 & Similar to Sed1 highly expressed in the stationary phase \\
\hline YFR044C & 4.3 & Similarity to the hypothetical protein YBR281C \\
\hline
\end{tabular}

The genes related to the transport system are underlined and the genes related to cold shock are indicated by broken lines. 
are underlined and account for about $50 \%$ of the top 25 genes. Thus, it may be suggested that $40 \mathrm{MPa}$ nitrogen gas affects cellular transport. These characteristics listed in Table 2 are different from those of hydrostatic pressure treatment because hydrostatic pressure induces genes linked to membrane metabolism such as INOI, OPI3, and RTAI (data not shown). High-pressure nitrogen gas is considered to be anaerobic and thus the effect on mitochondria only reflects the effect of gas pressure on transport in the mitochondria.

It has been reported that hydrostatic pressure induces genes which respond to heat shock proteins (13), but $40 \mathrm{MPa}$ nitrogen gas pressure did not induce heat shock proteins. In contrast, nitrogen gas pressure induced genes which respond to cold shock, such as TIRI and TIR2. These genes are shown in Table 2 and are indicated by broken lines. It is interesting to note that hydrostatic pressure apparently increased temperature, whereas gas pressure apparently decreased temperature.

We could observe living cells with 50\% colony-forming units and we could detect the responses after nitrogen gas treatment under $40 \mathrm{MPa}$. This suggests that, up to 40 $\mathrm{MPa}$, we can expect a response and we can characterize it. Generally other gases have a more toxic potential than nitrogen gas. The gas bioassay under high pressure can be applied to other gases to characterize the toxicity that causes damage to east cells.

\section{Acknowledgments}

We thank Dr. Randeep Rakwal for help in improving the manuscript.

\section{References}

1. Kohrer K \& Domdey H (1991). Preparation of high molecular weight RNA. In: Guthrie C \& Fink GR (Editors), Methods in Enzymology: Guide to Yeast Genetics and Molecular Biology. Academic Press, San Diego, CA, USA, 398-405.

2. Momose $Y$ \& Iwahashi $H$ (2001). Bioassay of cadmium using a DNA microarray: genome-wide expression patterns of Saccharomyces cerevisiae response to cadmium. Environmental Toxicology and Chemistry, 20: 2353-2360.

3. Ohmine K, Ota J, Ueda M et al. (2001). Characterization of stage progression in chronic myeloid leukemia by DNA microarray with purified hematopoietic stem cells. Oncogene, 20: 8249-8257.

4. Murata $\mathrm{Y}$, Momose $\mathrm{Y}$, Hasegawa M et al. (2002). Cluster analysis and display of genome-wide expression profiles in dimethyl sulfoxide treatment. Chem-Bio Informatics Journal, 2: 18-31.

5. Kurita S, Kitagawa E, Kim CH et al. (2002). Studies on the antimicrobial mechanisms of capsaicin using yeast DNA microarray. Bioscience, Biotechnology, and Biochemistry, 66: 532-536.

6. Kitagawa E, Takahashi J, Momose $Y$ et al. (2002). Effects of the pesticide thiuram: genome-wide screening of indicator genes by yeast DNA microarray. Environmental Science and Technology, 36: 3908-3915.

7. Murata Y, Watanabe T, Sato M et al. (2003). Dimethyl sulfoxide exposure facilitates phospholipid biosynthesis and cellular mem- brane proliferation in yeast cells. Journal of Biological Chemistry, 278: 33185-33193.

8. Kitagawa E, Momose $Y$ \& Iwahashi $H$ (2003). Correlation of the structures of agricultural fungicides to gene expression in Saccharomyces cerevisiae upon exposure to toxic doses. Environmental Science and Technology, 37: 2788-2793.

9. Sirisattha S, Momose Y, Kitagawa E et al. (2004). Toxicity of anionic detergents determined by Saccharomyces cerevisiae microarray analysis. Water Research, 38: 61-70.

10. Kim HJ, Ishidou E, Kitagawa E et al. (2004). A yeast DNA microarray for the evaluation of toxicity in environmental water containing burned ash. Environmental Monitoring and Assessment, 92: 253272

11. Parveen M, Hasan MK, Takahashi J et al. (2004). Response of Saccharomyces cerevisiae to a monoterpene: evaluation of antifungal potential by DNA microarray analysis. Journal of Antimicrobial Chemotherapy, 54: 46-55.

12. Mizukami S, Suzuki $Y$, Kitagawa E et al. (2004). Standardization of cDNA microarray technology for toxicogenomics; essential data for initiating cDNA microarray. Chem-Bio Informatics Journal, 4: 38-55.

13. Iwahashi $\mathrm{H}$, Shimizu H, Odani M et al. (2003). Piezophysiology of genome wide gene expression levels in the yeast Saccharomyces cerevisiae. Extremophiles, 7: 291-298. 